\title{
FINGERING CONVECTION IN ACCRETING HYDROGEN WHITE DWARFS
}

\author{
F.C. Wachlin ${ }^{1}$, S. Vauclair ${ }^{2}$, G. Vauclair ${ }^{3}$ and L.G. Althaus ${ }^{4}$
}

\begin{abstract}
The accretion of heavy material from debris disk on the surface of hydrogen-rich white dwarfs induces a double diffusivity instability known as the fingering convection. It leads to an efficient extra mixing which brings the accreted material deeper in the star than by considering only mixing in the surface dynamical convection zone, in a time scale much shorter than that of gravitational settling. We performed numerical simulations of a continuous accretion of heavy material having a bulk Earth composition on the two well studied DAZ and ZZ Ceti pulsators GD 133 and G 29-38. We find that the existence of fingering convection implies much larger accretion rates to explain the observed abundances than previous estimates based on the standard mixing length theory and gravitational settling only.
\end{abstract}

\section{Introduction: The fate of planetary systems}

What happens to planetary systems when their central star reaches the final evolutionary stage as a white dwarf? The study of the white dwarfs that exhibit debris disks and photospheric composition polluted by heavy elements may provide crucial informations on their formation, evolution and final fate. An increasing number of white dwarf stars are found to be surrounded by debris disks resulting from the tidal disruption of small rocky planetesimals which have survived

${ }^{1}$ Instituto de Astrofísica de La Plata,(UNLP,CONICET). Facultad de Ciencias Astronómicas y Geofísicas, Universidad Nacional de La Plata, Argentina

${ }^{2}$ Université de Toulouse, UPS-OMP, IRAP, France and CNRS, IRAP, 14 avenue Edouard Belin, 31400 Toulouse, France

${ }^{3}$ Université de Toulouse, UPS-OMP, IRAP, France and CNRS, IRAP, 14 avenue Edouard Belin, 31400 Toulouse, France

${ }^{4}$ Instituto de Astrofísica de La Plata,(UNLP,CONICET). Facultad de Ciencias Astronómicas y geofísicas, Universidad Nacional de La Plata, Argentina 
the whole evolutionary phases of the star (Jura 2003). Part of the matter of the disk falls onto the white dwarf surface, polluting its chemical composition. The accreted heavy elements do not stay at the stellar surface. They are mixed in the star outer layers through the fingering convection induced by the inverse $\mu$-gradient built by the accretion of heavy material, a process that we discussed in this paper. They also diffuse downwards by gravitational settling through the bottom of the mixed layers. The presence of heavy elements in white dwarf photosphere proves that the accretion process is going on. Estimates of the accretion rates and of the mass of the disrupted bodies have been determined previously by assuming that the observed heavy elements abundances reflect the equilibrium between the accretion and the gravitational settling rates (Farihi et al. 2012; Koester et al. 2014). However, these estimates did not take into account the extra-mixing induced by the fingering convection (refeered to as thermohaline convection in the oceanography context) resulting from the building of an inverse $\mu$-gradient. Deal et al. (2013) have explored the effects of the fingering convection in estimating the accretion rates on white dwarfs. We show here on the cases of the two well studied DAZ G29-38 and GD 133 how important is the extra-mixing induced by fingering convection and estimate new accretion rates.

\section{The fingering (thermohaline) convection}

The accreted matter has a chemical composition similar to the Earth bulk composition. Its mean molecular weight, $\mu$, is larger than the $\mu$ of the DA white dwarf underlying surface layers composed of pure $\mathrm{H}$. That is a situation characteristic of the fingering instability. It induces a turbulent zone which mixes the accreted matter much deeper than by considering only the mixing in the dynamical convection zone. Such an instability occurs in different astrophysical contexts where an inverse $\mu$-gradient is built (Vauclair 2004; Stancliffe et al. 2007; Stancliffe \& Glebbeek 2008; Thompson et al. 2008; Théado et al. 2009; Garaud 2011; Traxler et al. 2011; Wachlin et al. 2011; Théado \& Vauclair 2012; Vauclair \& Théado 2012; Brown et al. 2013; Wachlin et al. 2014; Zemskova et al. 2014; Deal et al. 2015).

\section{The accretion rates revisited}

G29-38 and GD 133 are two well studied polluted DA white dwarfs. Their photosphere contains a number of heavy elements with precisely determined abundances (Xu et al. 2014). Simulations of a continuous accretion of matter on models of G29-38 and GD 133 have been performed. The chemical composition of the accreted matter is similar to the Earth bulk composition. Our simulations were done in the framework of the double-diffusive theory developed by Grossman et al. (1993). The results are compared with those obtained when one uses the classical mixing length theory for convection (ML2, with $\alpha=0.8$ ). Figure 1 illustrates how 


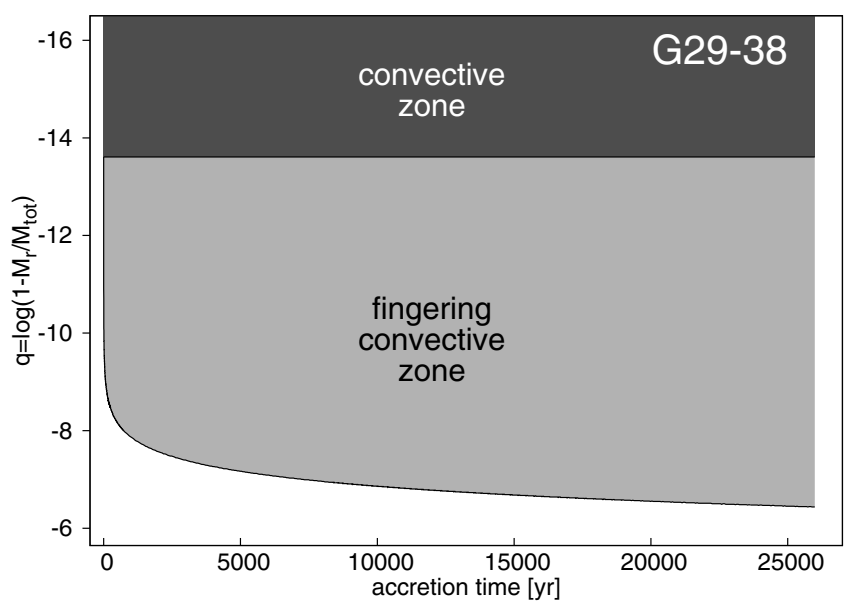

Fig. 1. Time evolution of the stellar structure for the G29-38 model. The logarithm of the mass fraction is plotted as a function of time after the accretion process started at $t=0$, for an accretion rate of $10^{10} \mathrm{gs}^{-1}$. The region mixed by the fingering convection develops rapidly once the accretion starts. It reaches a mass fraction about 7 orders of magnitude larger than the dynamical convection zone after 25000 years where our numerical simulation stops.

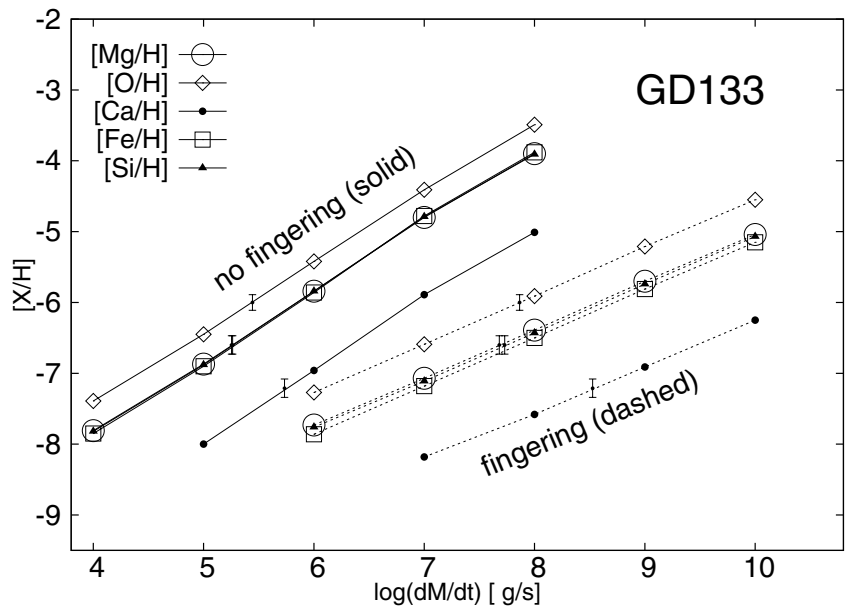

Fig. 2. Logarithmic relative surface abundances of $\mathrm{Mg}, \mathrm{O}, \mathrm{Ca}, \mathrm{Fe}$ and $\mathrm{Si}$ as a function of the accretion rates for GD 133; the error bars are the abundances determined from observations (Xu et al. 2014); when fingering convection is taken into account, accretion rates 100 or 1000 larger than those estimated when the classical mixing length theory and gravitational settling are only considered are needed to obtain the same surface abundances. 


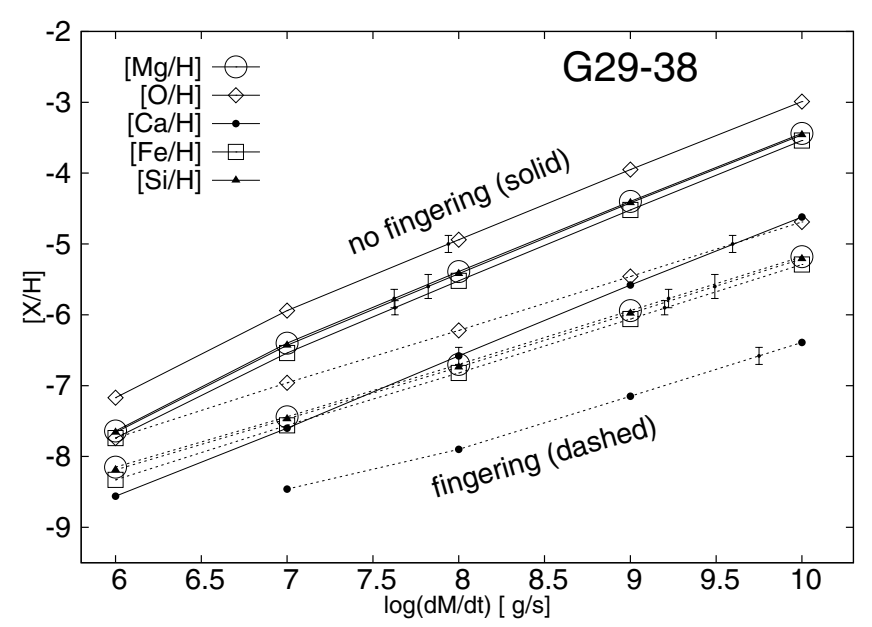

Fig. 3. Same as Figure 2 for G29-38. The accretion rate needed to reproduce the observed abundances is 10 to 100 larger than estimated from mixing length theory and gravitational settling when fingering convection is taken into account.

the fingering convection starts below the dynamical convection zone as soon as the accretion proceeds, and evolves with time, in the case of an accretion rate of $\log \left(\mathrm{d} M_{a c c} / \mathrm{dt}\right)[\mathrm{g} / \mathrm{s}]=10$ on $\mathrm{G} 29-38$. The fractional mass mixed in the fingering convection zone exceeds by about seven orders of magnitude the fractional mass of the dynamical convection zone after 25000 years of accretion. For our simulations, we considered a range of accretion rates, $\log \left(\mathrm{d} M_{a c c} / \mathrm{dt}\right)[\mathrm{g} / \mathrm{s}]$, from 4 to 10 . The surface abundance for $\mathrm{O}, \mathrm{Mg}, \mathrm{Si}, \mathrm{Ca}$ and $\mathrm{Fe}$ were computed as a function of time in the two cases: 1) without taking fingering convection into account and 2) taking fingering convection into account. In both cases the surface abundances reach a stationary value. In case 2 the accreted matter is mixed much deeper due to the fingering convection and it takes longer to reach the stationary abundances. Figures 2 and 3 show the stationary abundances obtained in the two cases as a function of the accretion rates for GD 133 and G29-38 respectively. The most important result is that when fingering convection is properly taken into account, it requires accretion rates larger by 1 to 2 orders of magnitude for G29-38 and by 2 to 3 orders of magnitude for GD 133 than when fingering convection is ignored, to reproduce the same surface abundances.

Our results show that it is important to take the fingering convection into account in order to get realistic estimates of the accretion rates of debris disks material on hydrogen-atmosphere white dwarfs. 


\section{References}

Brown, J.M., Garaud, P., \& Stellmach, S., 2013, ApJ, 768, 34

Deal, M., Deheuvels, S., Vauclair, G., Vauclair, S., \& Wachlin, F.C., 2013, A\&A, 557, L12

Deal, M., Richard, O., \& Vauclair, S., 2015, A\&A, 584, 105

Farihi, J., Gänsicke, B.T., Wyatt, M.C., et al., 2012, MNRAS, 424, 464

Garaud, P., 2011, ApJ, 728, L30

Grossman, S.A., Narayan, R., \& Arnett, D., 1993, ApJ, 407, 284

Jura, M., 2003, ApJ, 584, L91

Koester, G., Gänsicke, B.T., \& Farihi, J., 2014, A\&A, 566, 34

Stancliffe, R.J., \& Glebbeek, E., 2008, MNRAS, 389, 1828

Stancliffe, R.J., Glebbeek, E., Izzard, R.G., \& Pols, O., 2007, A\&A, 464, L57

Théado, S. \& Vauclair, S., 2012, ApJ, 744, 123

Théado, S., Vauclair, S., Alecian, G., \& LeBlanc, F., 2009, ApJ, 704, 126

Thompson, I.B., Ivans, I.I., Bisterzo, S. et al., 2008, ApJ, 677, 556

Traxler, A., Garaud, P., \& Stellmach, S., 2011, ApJ, 728, L29

Vauclair, S., 2004, ApJ, 605, 874

Vauclair, S., \& Théado, S., 2012, ApJ, 753, 49

Wachlin, F.C., Miller Bertolami, M.M., \& Althaus, L.G., 2011, A\&A, 533, 139

Wachlin, F.C., Vauclair, S. \& Althaus, L.G., 2014, A\&A, 570, 58

Xu, S., Jura, M., Koester, D., Klein, B., \& Zuckerman, B., 2014, ApJ, 795, 118

Zemskova, V., Garaud, P., Deal, M., \& Vauclair, S., 2014, ApJ, 795, 118 